\title{
Identification of biomarkers in macrophages of atherosclerosis by microarray analysis
}

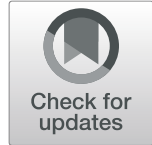

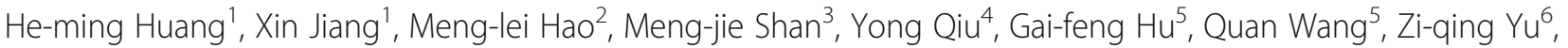
Ling-bing Meng ${ }^{7 *+}$ and Yun-yun Zou ${ }^{8^{*}+}$

\begin{abstract}
Background: Atherosclerotic cardiovascular disease (ASCVD) refers to a series of diseases caused by atherosclerosis (AS). It is one of the most important causes of death worldwide. According to the inflammatory response theory, macrophages play a critical role in AS. However, the potential targets associated with macrophages in the development of AS are still obscure. This study aimed to use bioinformatics tools for screening and identifying molecular targets in AS macrophages.

Methods: Two expression profiling datasets (GSE7074 and GSE9874) were obtained from the Gene Expression Omnibus dataset, and differentially expressed genes (DEGs) between non-AS macrophages and AS macrophages were identified. Functional annotation of the DEGs was performed by analyzing the Gene Ontology and Kyoto Encyclopedia of Genes and Genomes databases. STRING and Cytoscape were employed for constructing a protein-protein interaction network and analyzing hub genes.

Results: A total of 98 DEGs were distinguished between non-AS macrophages and AS macrophages. The functional variations in DEGs were mainly enriched in response to hypoxia, respiratory gaseous exchange, protein binding, and intracellular, ciliary tip, early endosome membrane, and Lys63-specific deubiquitinase activities. Three genes were identified as hub genes, including KDELR3, CD55, and DYNC2H1.
\end{abstract}

Conclusion: Hub genes and DEGs identified by using microarray techniques can be used as diagnostic and therapeutic biomarkers for AS.

Keywords: Atherosclerotic cardiovascular disease, Atherosclerosis, Macrophages, Hub genes, Bioinformatics

\section{Background}

Atherosclerotic cardiovascular disease (ASCVD) refers to a series of diseases caused by atherosclerosis (AS), including acute coronary syndromes, myocardial infarction, stable and unstable angina, revascularization of coronary or other arterial blood vessels, stroke, transient ischemic attack, and arterial AS sclerosing peripheral arterial disease [1]. ASCVD is one of the most important causes of death in elderly patients at home and abroad [2]. According to the World Health Organization, 17 million people died of ASCVD worldwide in 2008, accounting for $30 \%$ of the total disease mortality, $80 \%$ of which occurred in low- and

\footnotetext{
* Correspondence: 314597690@qq.com; 1048482456@qq.com

†Ling-bing Meng and Yun-yun Zou contributed equally to this work.

${ }^{7}$ Neurology Department, Beijing Hospital, National Center of Gerontology,

No.1 Dahua Road, Dong Dan, Beijing 100730, People's Republic of China

${ }^{8}$ The Fifth Ward of Ophthalmology Department, Shenzhen Eye Hospital,

Shenzhen 518040, People's Republic of China

Full list of author information is available at the end of the article
}

middle-income countries. It is predicted that, by 2030, the number of deaths due to ASCVD will increase to 23.3 million. The burden of ASCVD is increasing, and the disease has become a major public health problem. It is, therefore, particularly important to strengthen current research mechanisms for this disease [3].

AS is an arterial disease associated with dyslipidemia and changes in the components of blood vessel walls. It mainly affects large and medium-sized arteries-especially those in the heart, brain, kidneys, and other organs-and can cause ischemic changes [4]. The currently recognized risk factors for AS include smoking, alcohol, genetic factors, hypertension, and hyperlipidemia. Some diseases that cause secondary hyperlipidemia (e.g., diabetes and nephrotic syndrome) and other factors (e.g., age and obesity) [5]. The pathogenesis of AS has been a hot topic in the medical world. For a long time in the past, AS was considered as passive fat deposition in blood vessel walls. We

(c) The Author(s). 2019 Open Access This article is distributed under the terms of the Creative Commons Attribution 4.0 International License (http://creativecommons.org/licenses/by/4.0/), which permits unrestricted use, distribution, and 
now know that AS is a chronic inflammatory disease caused by lipids, especially low-density lipoproteins (LDLs), and white blood cells. Increased plaque inflammatory response can induce local protein hydrolysis, resulting in plaque rupture and thrombosis, ultimately leading to the development of ASCVD [6]. This phenomenon formed the basis for the "inflammatory response theory".

Regarding the theory of inflammatory response, the statement by Ross [7] that "AS is an inflammatory disease" is recognized by most scholars. The development of AS lesions is mainly influenced by network relationships between endothelial cells, smooth muscle cells, macrophages, and $\mathrm{T}$ lymphocytes. Macrophage production leads to the secretion of several growth-stimulating factors, which cause changes in the phenotype of blood vessels. The phenotype of smooth muscle cells changes from their original normal contractile type to the naive synthesis type; these cells then proliferate, migrate to the endometrium, synthesize and secrete stimulating factors, and finally stimulate macrophages, leading to continuous macrophage proliferation. T lymphocytes continue to proliferate and replicate like macrophages. Macrophages and T lymphocytes play an important role in AS. Therefore, reducing cholesterol accumulation in macrophages and inhibiting vascular wall inflammation are important for the prevention and treatment of AS [8].

An increasing number of studies have shown that the role of macrophages in the development of AS is closely related to mutation and abnormal expression of genes [9-11]. Higashi et al. indicated that the macrophage insulin-like growth factor-1 receptor signaling pathway inhibits the aggregation of macrophages and foam cells during the development of atherosclerotic lesions and reduces plaque instability, providing a novel mechanism for antiatherogenic research [9]. In addition, Wang et al. demonstrated the critical role of C1q/TNF-related proteins-1 (CTRP1) in linking the dysregulation of lipid metabolism and inflammatory responses in macrophages. Stimulation of CTRP1 significantly enhances the secretion of proatherogenic factors, including monocyte chemoattractant protein-1, tumor necrosis factor- $\alpha$ (TNF- $\alpha$ ), interleukin-1 beta, and interleukin6 (IL-6) [10]. Moreover, another study has suggested that deletion or low expression of interferon regulatory factor 2-binding protein 2 (IRF2BP2) in macrophages can aggravate AS [11]. However, because of the lack of strategies for timely detection, dynamic monitoring, and effective control of macrophages, it has not been possible to effectively alleviate atherosclerotic cardiovascular and cerebrovascular diseases worldwide. Therefore, it is particularly important to identify the precise genetic targets associated with macrophages in the development of AS [12].

With the rapid development of science and technology in the past few years, bioinformatics and microarray technologies have become widely employed for screening and predicting disease gene targets [13]. These technologies can help researchers identify differentially expressed genes (DEGs) and potentially different pathways between non-AS and AS macrophages.

This study aimed to employ bioinformatics tools for screening and identifying molecular targets in AS macrophages. Two human gene expression profiling datasets were downloaded from the Gene Expression Omnibus (GEO) dataset and analyzed in order to identify DEGs between non-AS and AS macrophages. Then, the molecular mechanisms underlying the development of AS macrophages were researched by enrichment and protein-protein interaction (PPI) network analyses. In the end, 98 DEGs and 3 hub genes were conclusively authenticated.

\section{Methods}

\section{Access to public-domain data}

GEO (http://www.ncbi.nlm.nih.gov/geo) is an open functional genomics database of high-throughput resources, including data from microarray, gene expression, and gene chip analyses. For this study, we downloaded two expression profiling datasets (GSE7074 and GSE9874) from GEO. The probes were transformed into homologous gene symbols by using the platform's annotation data. All macrophage data were derived from in silico studies. The GSE7074 dataset contains 8 non-AS-macrophage samples from non-atherosclerotic tissues and 8 AS-macrophage samples from atherosclerotic plaques of the carotid artery. The GSE9874 dataset contains 15 non-AS-macrophage samples from subjects without AS and 15 AS-macrophage samples from atherosclerotic tissues.

\section{DEGs identified by GEO2R}

GEO2R (http://www.ncbi.nlm.nih.gov/geo/geo2r), an online data analysis tool, was used for screening DEGs between non-AS- and AS-macrophage samples. After setting up differential experimental groups for one of the GEO series, GEO2R was instructed to execute a command for comparing differential classifications in order to identify DEGs. When the gene symbol corresponded to the probes, the data were usually considered valuable and were reserved. The level of statistical significance was set at $P \leq 0.05$.

\section{Functional annotation of DEGs by GO and KEGG analysis}

DAVID (https://david.ncifcrf.gov/home.jsp; version 6.8), an online analysis tool suite for integrated discovery and annotation, mainly provides typical batch annotation and gene-GO term enrichment analysis for highlighting the most relevant GO terms associated with a given gene list. Kyoto Encyclopedia of Genes and Genomes (KEGG) (https://www.kegg.jp/), one of the most commonly used biological information databases in the world, aims to 
understand advanced functions in biological systems. At a molecular level, KEGG specifically integrates a large number of practical program database resources from high-throughput experimental technologies. Gene Ontology (GO) is a widely used ontology in bioinformatics; it covers three aspects of biology: cellular component (CC), molecular function (MF), and biological process (BP). In this study, the DAVID online tool was used to perform GO and KEGG analysis of the DEGs. The level of statistical significance was set at $P<0.05$.

\section{Construction and analysis of a PPI network}

The common DEGs were imported to the online "Search Tool for the Retrieval of Interacting Genes" (STRING; http://string-db.org; version 10.5), which predicted and traced the PPI network. Analysis of interactions between various proteins might provide some novel ideas on the pathophysiological mechanisms of AS development. In this study, a PPI network of DEGs was constructed by using the STRING database with a minimum required interaction score of $>0.4$ (medium confidence).

\section{Mining and analysis of hub genes}

Molecular Complex Detection (MCODE; version 1.5.1), a plugin of Cytoscape, can discover tightly coupled regions on the basis of topological principles. In this study, MCODE was used to identify the most important module of the PPI network map. The criteria for MCODE analysis were as follows: degree cutoff, 2; MCODE scores, > 5; max depth, 100; k-score, 2; and node score cutoff, 0.2. Hub genes were identified when the degree cutoff was set at $\geq 10$. Then, the hub genes were submitted for GO and clustering analysis by using OmicShare (http://www.omicshare.com/tools), an open data analysis platform.

\section{Results}

DEGs identified between non-AS- and AS-macrophages samples

After analyzing the GSE7074 and GSE9874 datasets by using GEO2R, the differences between non-AS- and AS-macrophage samples were plotted in the form of volcano plots (Figs. 1 a,b). Then, these results were standardized, and the DEGs between non-AS and AS macrophages (1860 in GSE7074 and 782 in GSE9874) were identified. A Venn diagram showed that the two datasets contained 98 DEGs in common (Fig. $1 \mathrm{c}$ ).

Functional annotation of DEGs by GO and KEGG analysis The results of $\mathrm{GO}$ analysis demonstrated that the variations in BPs, CCs, and MF of the DEGs were mainly enriched in negative regulation of TOR (target of rapamycin) signaling, response to hypoxia, respiratory gaseous exchange, thiol-dependentubiquitin-specific protease activity, protein binding, Lys63-specific deubiquitinase activity, and intracellular, ciliary tip, and early endosome membrane activities (Table 1). The results of analysis of KEGG pathways revealed that all DEGs were primarily enriched in the Ras, insulin, ErbB, and gonadotropin-releasing hormone (GnRH) signaling pathways (Table 1).

\section{PPI network construction and module analysis}

A PPI network of the DEGs was constructed (Fig. 2), and the most significant module of the PPI network was identified by using Cytoscape (Fig. 3). Functional analysis of the genes involved in this module was performed by using DAVID. The results showed that the genes in this module were mainly involved in biological regulation, cellular processes, response to stimulus, signaling, cells, cell parts, organelles, extracellular regions, membranes, macromolecular complexes, binding, catalytic activity, and molecular function regulation (Fig. 4).

\section{Hub gene selection and analysis}

A total of three genes-KDELR3 (KDEL endoplasmic reticulum protein retention receptor 3), CD55 (complement decay-accelerating factor), and $\mathrm{DYNC2H1}$ (dynein cytoplasmic 2 heavy chain 1) - were identified 'as hub genes from the most significant module, with a degree cutoff of $\geq 10$. The names, abbreviations, and functions of these hub genes are shown in Table 2. The results of hierarchical clustering showed that these hub genes could differentiate AS-macrophage samples from non-AS-macrophages samples (Figs. 5 a,b). These hub genes showed the highest node score in the PPI network, suggesting that they might play important roles in the occurrence or progression of AS.

\section{Discussion}

The morbidity and mortality of AS have increased over the years, seriously endangering human health [14]. The pathogenesis of AS is complex and explained by many theories, including "lipid infiltration" and "inflammatory response" [15]. On the one hand, very-low-density lipoproteins, LDLs, and lipoproteins invade and accumulate in the vascular wall, resulting in the thickening of the vascular intima and narrowing of the lumen. Monocytes differentiate into macrophages and phagocytose a large amount of lipids to transform into foam cells, promoting the development of AS [16]. On the other hand, foam cells secrete several inflammatory factors-such as TNF- $\alpha$, interleukin1 (IL-1), and IL-6-which further leads to cellular adhesion, infiltration of inflammatory cells, and matrix degradation, resulting in atherosclerotic plaque rupture [15]. Therefore, inhibiting the accumulation of lipids in macrophages and decreasing inflammatory response are critical measures for preventing AS development. Metabolic processes in macrophages are affected by multiple target genes, and a single target gene probably also 

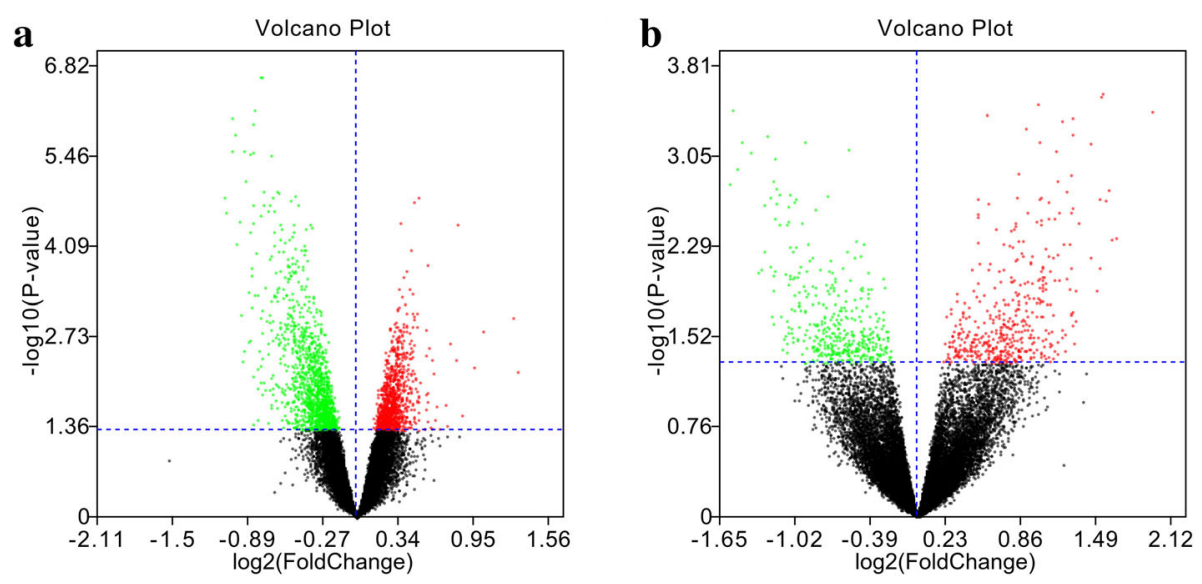

\section{c}

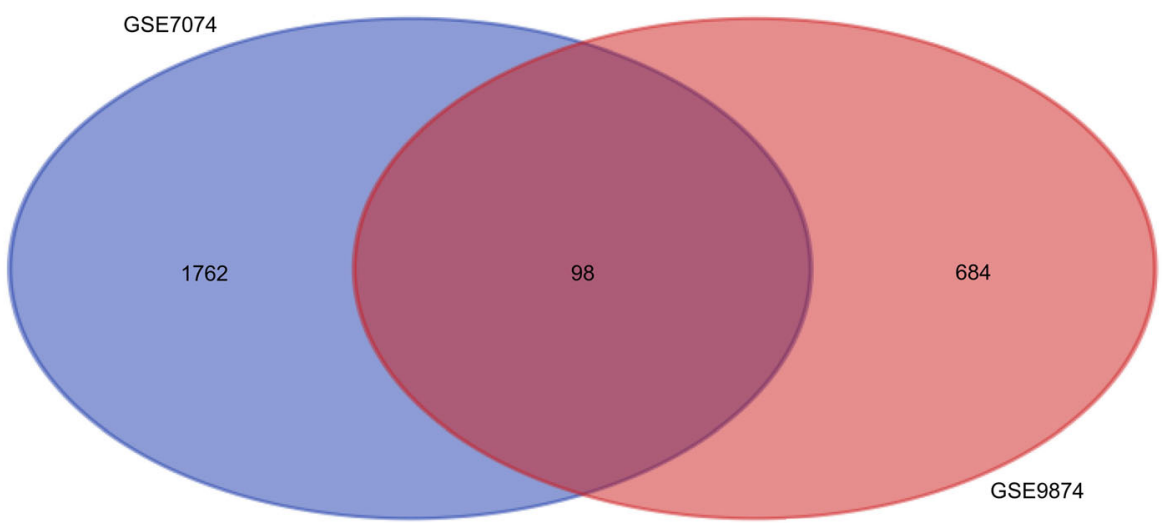

Fig. 1 Identification of DEGs between non-AS and AS macrophages. Volcano plots presenting the differences between non-AS and AS macrophages after analysis of the (a) GSE7074 and (b) GSE9874 datasets by using GEO2R; (c) Venn diagram showing the 98 DEGs identified simultaneously in the GSE7074 and GSE9874 datasets. DEG: differentially expressed genes; AS, atherosclerosis

Table 1 GO and KEGG pathway enrichment analysis of DEGs between non-AS macrophages and AS macrophages

\begin{tabular}{|c|c|c|c|}
\hline Term & Description & Count in gene set & $P$-value \\
\hline GO:0032007 & negative regulation of TOR signaling & 3 & 0.010 \\
\hline GO:0001666 & response to hypoxia & 5 & 0.014 \\
\hline GO:0007585 & respiratory gaseous exchange & 3 & 0.015 \\
\hline GO:0005622 & intracellular & 16 & 0.003 \\
\hline GO:0097542 & ciliary tip & 3 & 0.022 \\
\hline GO:0031901 & early endosome membrane & 4 & 0.022 \\
\hline GO:0004843 & thiol-dependent ubiquitin-specific protease activity & 4 & 0.009 \\
\hline GO:0005515 & protein binding & 58 & 0.015 \\
\hline GO:0061578 & Lys63-specific deubiquitinase activity & 2 & 0.026 \\
\hline hsa04014 & Ras signaling pathway & 7 & 0.003 \\
\hline hsa04910 & Insulin signaling pathway & 5 & 0.012 \\
\hline hsa04012 & ErbB signaling pathway & 4 & 0.019 \\
\hline hsa04912 & GnRH signaling pathway & 4 & 0.021 \\
\hline
\end{tabular}




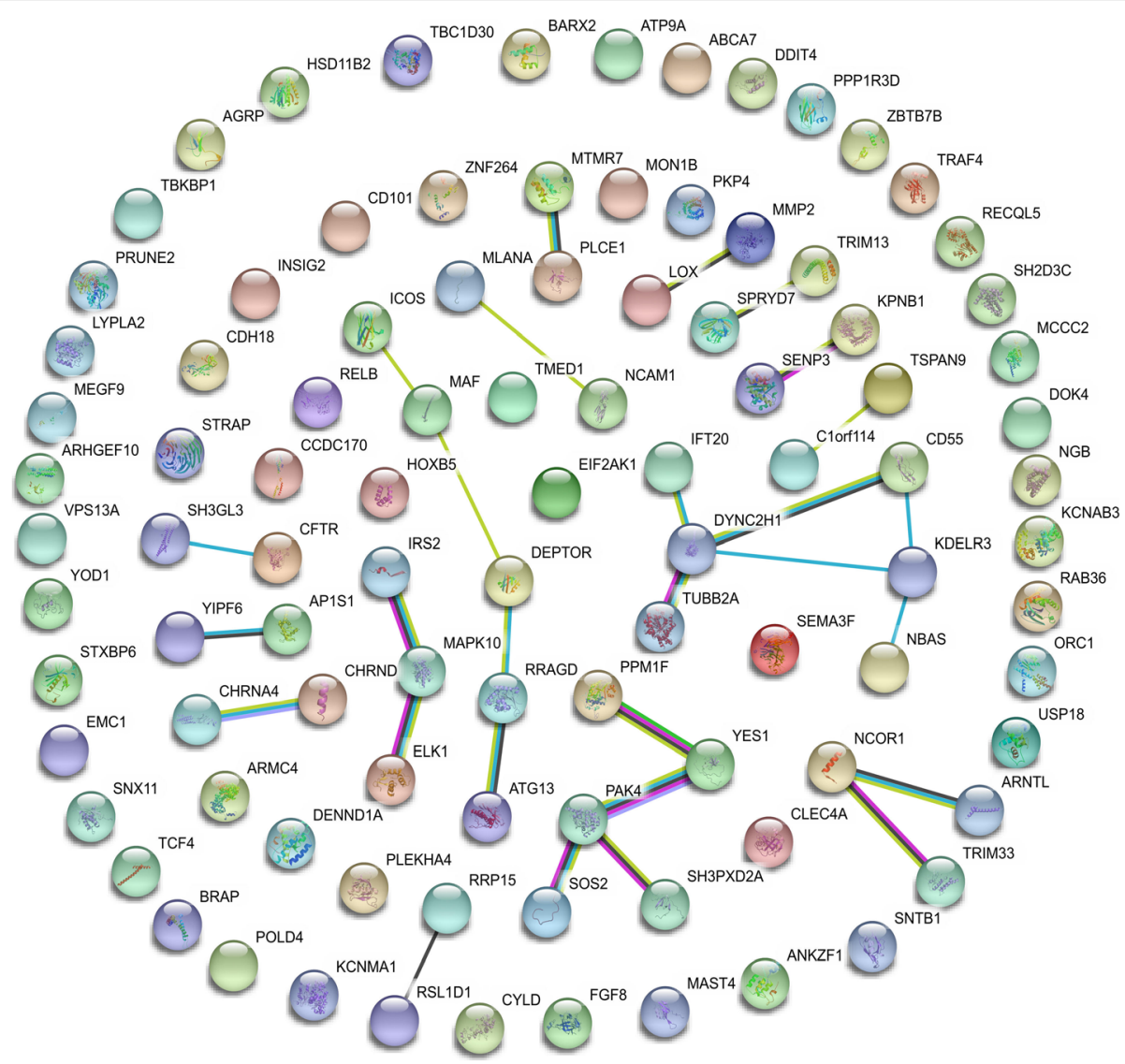

Fig. 2 Protein-protein interaction network of differentially expressed genes

regulates multiple micro-RNAs, which makes research on the occurrence and development of AS macrophages complex and challenging $[17,18]$. It is, therefore, critical to explore the precise molecular mechanism of macrophages in AS and identify reliable therapeutic targets.

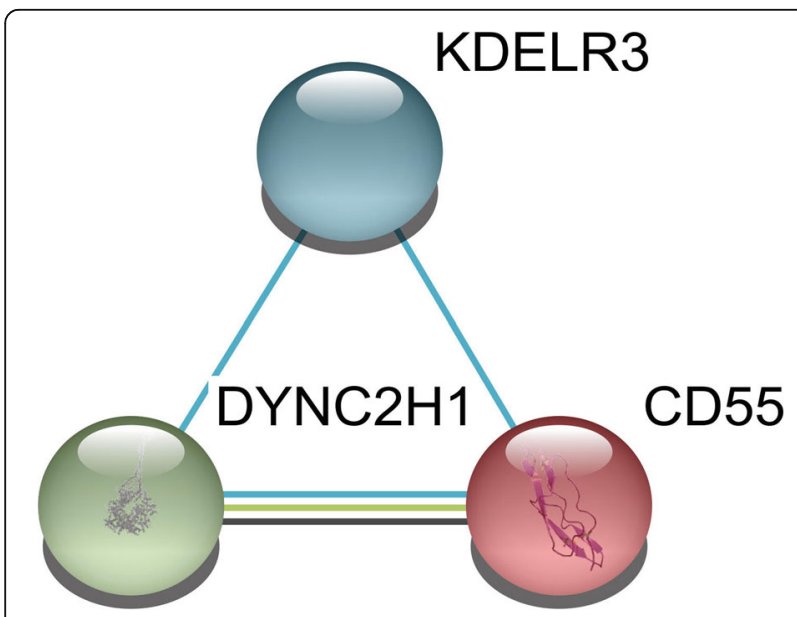

Fig. 3 The most significant module identified in the protein-protein interaction network
With recent developments in biogenetics exploration technologies, studies have demonstrated differential gene expression in several other diseases, which provides ideas for accurate treatment of such diseases [19-21]. For example, four molecular markers associated with the development of gliomas have been identified through biogenetics approaches [19]. A study on hepatocellular carcinoma employed microarray technology to search and analyze a biological database and finally identified 16 hub genes closely related to the development of hepatocellular carcinoma [20]. Another study analyzed the expression levels of 322 immune genes and found that the immune characteristics of patients with neuroblastoma were enhanced; this study finally selected eight immune genes as molecular markers of neuroblastoma [21]. The emergence of biogenetics techniques and their application in prediction of disease target genes provide us new directions and technical support for selecting DEGs related to AS macrophages.

In this study, we used bioinformatics tools to identify DEGs and target molecular markers in AS macrophages. Analysis of the GSE7074 and GSE9874 datasets by using GEO2R helped us identify DEGs between non-AS- and AS-macrophage samples. Both datasets included 98 DEGs 


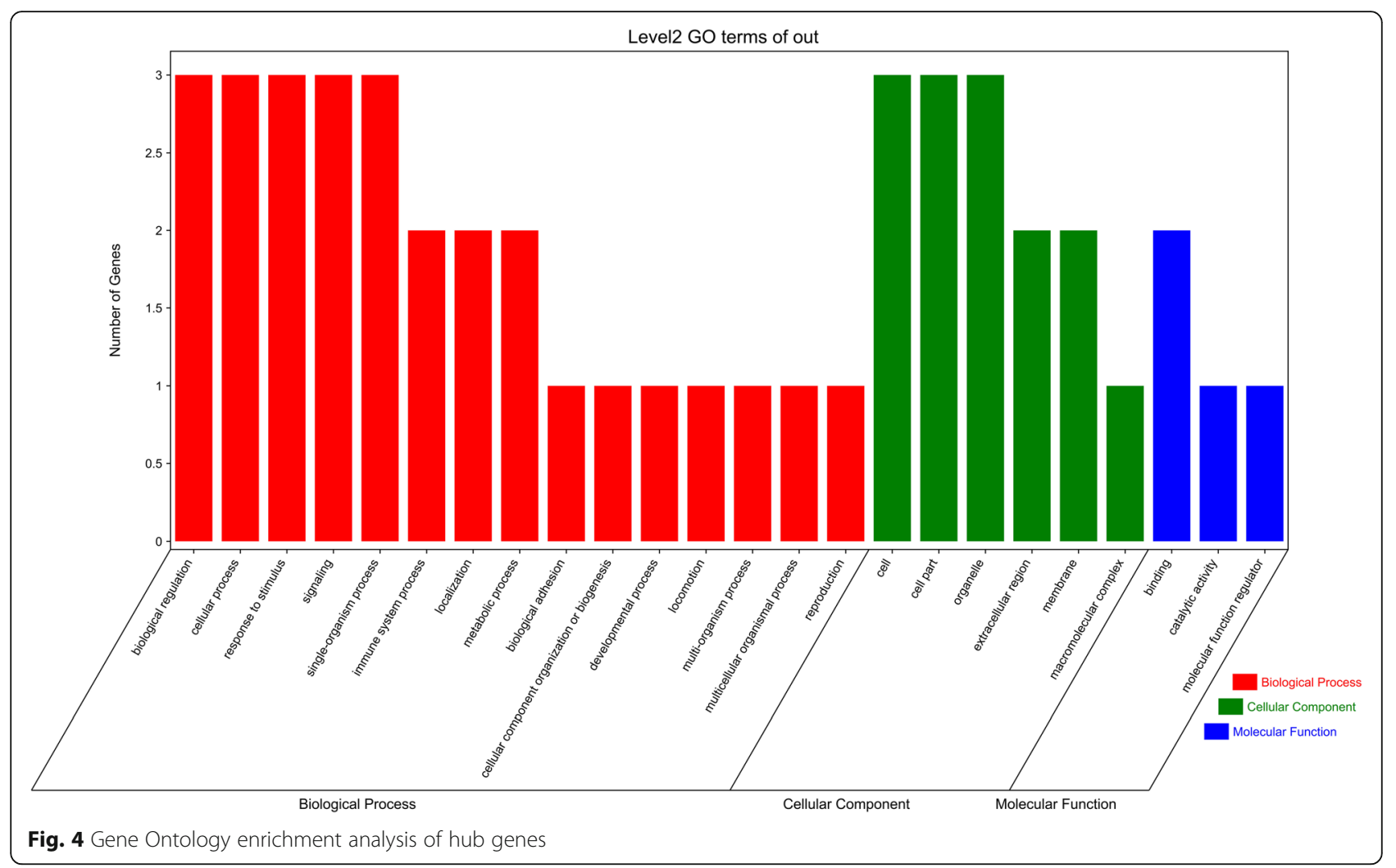

in common. Through enrichment analysis, we found that these DEGs were mainly enriched in negative regulation of TOR signaling; response to hypoxia; respiratory gaseous exchange; intracellular, ciliary tip, and early endosome membrane activities; thiol-dependent ubiquitin-specific protease activity; protein binding; Lys63-specific deubiquitinase activity; and the Ras, insulin, ErbB, and GnRH signaling pathways. Furthermore, in the most significant module of the PPI network, three hub genes-KDELR3, $C D 55$, and $D Y N C 2 H 1$ - showed the highest score, suggesting that they might have significant roles in the occurrence or development of AS.

KDELR3, the third confirmed member of the KDEL family, encodes proteins associated with the endoplasmic reticulum (ER) [22]. According to our analysis, KDELR3 expression in AS macrophages was significantly different from that in non-AS tissues. KDELR3 can regulate the specific functions of proteins in the ER lumen. However, the ER is the center for the synthesis of important biomacromolecules such as proteins, lipids (e.g., triglycerides), sugars, and nucleic acids. The ER exists in all types of eukaryotic cells except mature mammalian erythrocytes [23]. It is an interconnected lamellar lacunar or tubular system composed of biofilms. The lacunar space between the membranes is called a pool, and it is usually not directly connected with the extracellular lacunae or cytoplasmic matrix. This intracellular membranous piping system constitutes the intracellular transport pathway for materials and also provides a broad reaction area for a variety of enzymes within the

Table 2 Summaries for the function of 3 hub genes

\begin{tabular}{llll}
\hline No. & Gene symbol & Full name & Function \\
\hline 1 & KDELR3 & KDEL endoplasmic reticulum & Required for the retention of luminal endoplasmic reticulum proteins. Determines the specificity of \\
& protein retention receptor 3 & $\begin{array}{l}\text { the luminal ER protein retention system. Also required for normal vesicular traffic through the } \\
\text { Golgi. This receptor recognizes K-D-E-L (By similarity). }\end{array}$
\end{tabular}

2 CD55 CD55 molecule (Cromer blood group)
This gene encodes a glycoprotein involved in the regulation of the complement cascade. Binding of the encoded protein to complement proteins accelerates their decay, thereby disrupting the cascade and preventing damage to host cells. Antigens present on this protein constitute the Cromer blood group system (CROM). Alternative splicing results in multiple transcript variants. The predominant transcript variant encodes a membrane-bound protein, but alternatively spliced transcripts may produce soluble proteins

3 DYNC2H1 Dynein cytoplasmic 2 May play a role in transport between endoplasmic reticulum and Golgi or organization of the Golgi in cells (By similarity). 

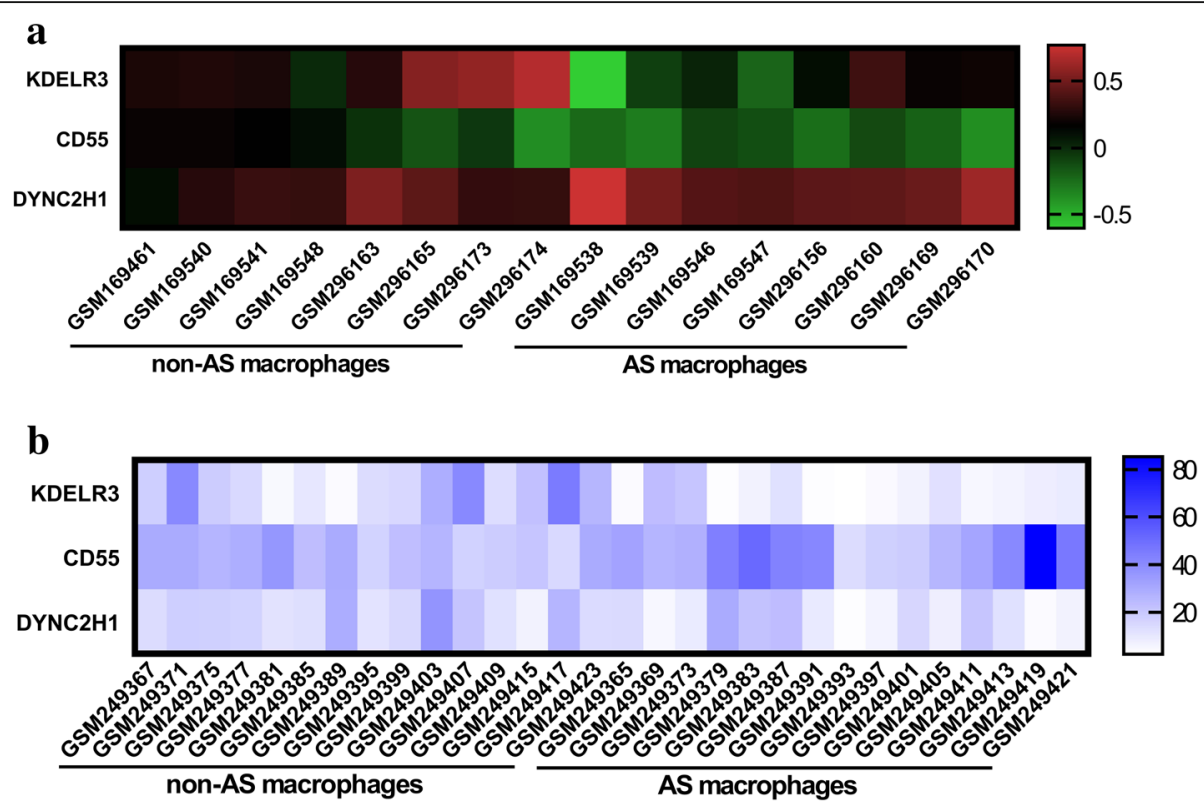

Fig. 5 Results of hierarchical clustering showing that the hub genes can differentiate AS and non-AS macrophages in the (a) GSE7074 and (b) GSE9874 datasets

cell [23]. The ER is connected with the nuclear and cell membranes and can be divided into smooth and rough ER. Smooth ER has a smooth surface and does not bind ribosomes. It is mainly involved in the synthesis and transportation of steroids and lipids as well as in glucose metabolism and hormone inactivation. Studies have shown that stress is one of the risk factors for AS development [24]. Macrophages activated after stress contain abundant smooth ER, which is involved in lipoprotein processing and modification and lipid synthesis and transport and thus participates in the formation and development of AS. Therefore, we speculate that KDELR3 expression plays an important role in the development of AS macrophages. Therefore, KDELR3 could serve as a diagnostic marker of AS.

CD55 encodes glycoproteins involved in the regulation of complement cascades, which bind to complement proteins and accelerate their own decay, a process that disrupts complement cascades and avoids killing host cells [25]. According to our results, CD55 expression in AS-macrophage samples was significantly lower than that in non-AS tissues. Complement proteins are a type of serum proteins found in the serum and tissue fluids of humans and vertebrates. They possess enzymatic activity and can mediate immune responses and inflammation after activation [26]. The complement system is activated through three relatively independent but interrelated pathways in order to exert a variety of biological effects, including regulation of phagocytosis, cell lysis, mediation of inflammation, immune regulation, and immune-complex clearance. The complement system can not only enhance phagocytosis and macrophage chemotaxis but also increase vascular permeability. Complement cascade is a process that amplifies weak biological signals and enhances their biological effects in a step-by-step manner [27]. We, therefore, speculate that CD55 is expressed at low concentrations in AS samples. When the body is stimulated by various endogenous and exogenous stimuli, the CD55-encoded glycoprotein fails to block the complement cascade, which results in the enhancement of macrophage chemotaxis and inflammatory response, further aggravating AS. We, therefore, speculate that CD55 expression plays an important role in the development of AS.

DYNC2H1 encodes a cytoplasmic dynamic protein which acts as a motor for the axoplasmic transport of cellular cilia and might play an important role in transport between the ER and Golgi apparatus [28]. According to our results, DYNC2H1 expression levels in AS-macrophage samples were significantly higher than those in non-AS tissues. As mentioned above, when the body is stimulated by stress, macrophages are activated, and a large amount of smooth ER accumulates in the cytoplasm, where it participates in the synthesis and transportation of steroids and lipids [29]. DYNC2H1 is expressed at high concentrations in AS samples; this DYNC2H1 upregulation enhances the transportation power of the ER and promotes lipid phagocytosis by macrophages, further advancing the progression of AS. Therefore, we speculate that $D Y N C 2 H 1$ expression plays an important role in the development of AS. Furthermore, $D Y N C 2 H 1$ may be used as a therapeutic target for AS in a clinical setting. 
The present study has some limitations. The screening of the three key genes related to the development and progression of AS was based on bioinformatics technologies and thus requires further validation in in vitro and in vivo experiments. Therefore, relevant samples should be collected in clinical work, and an animal model of AS should be established to verify the mechanism of action of the three genes in AS macrophages.

\section{Conclusions}

In conclusion, we employed bioinformatics and microarray techniques to identify 98 DEGs and 3 hub genes in AS and non-AS macrophages. These genes could serve as diagnostic and therapeutic biomarkers for AS.

\section{Abbreviations}

AS: Atherosclerosis; ASCVD: Atherosclerotic cardiovascular disease; BP: Biological process; CC: Cell component; CD55: Complement decay-accelerating factor; CTRP1: C1q/TNF-related proteins-1; DEG: Differentially expressed gene; DYNC2H1: Dynein cytoplasmic 2 heavy chain 1; ER: Endoplasmic reticulum; GEO: Gene Expression Omnibus; GnRH: Gonadotropin-releasing hormone; GO: Gene Ontology; IL-1: interleukin-1; IL-6: interleukin-6; IRF2BP2: Interferon regulatory factor 2-binding protein 2; KDELR3: KDEL endoplasmic reticulum protein retention receptor 3; KEGG: Kyoto Encyclopedia of Genes and Genomes; LDL: Low-density lipoprotein; MCODE: Molecular Complex Detection; MF: Molecular function; PPI: Protein-protein interaction; STRING: Search Tool for the Retrieval of Interacting Genes; TNF-a: Tumor necrosis factor-a; TOR: Target of rapamycin

\section{Acknowledgements}

We are thankful to Ya-Lun Dai (Epidemiology Department, Beijing Hospital, National Center of Gerontology) for providing statistical assistance and suggestions during the submission process.

\section{Funding}

Not applicable.

\section{Availability of data and materials}

The datasets used and/or analyzed during the current study are available from the corresponding author on reasonable request.

\section{Authors' contributions}

He-Ming Huang and Xin Jiang performed the experiments and were major contributors in the writing and submission of the manuscript. Ling-Bing Meng and Yun-Yun Zou made substantial contributions towards conceptualizing the study. Yong Qiu and Gai-Feng Hu designed the draft of the research process. Meng-Lei Hao and Meng-Jie Shan were involved in revising the manuscript critically for important intellectual content. Quan Wang and Zi-Qing Yu analyzed the animal data on atherosclerosis. All authors read and approved the final manuscript.

\section{Ethics approval and consent to participate}

Not applicable.

\section{Consent for publication}

Not applicable.

\section{Competing interests}

The authors declare that they have no competing interests.

\section{Publisher's Note}

Springer Nature remains neutral with regard to jurisdictional claims in published maps and institutional affiliations.

\section{Author details}

'Geriatric Department, Shenzhen People's Hospital, The Second Clinical Medical College of Jinan University, The First Affiliated Hospital of Southern University of Science and Technology, Shenzhen 518020, People's Republic of China. ${ }^{2}$ Department of Geriatric Medicine, Qinghai University, Xining, Qinghai 810016, People's Republic of China. ${ }^{3} \mathrm{MOH}$ Key Laboratory of Systems Biology of Pathogens, Institute of Pathogen Biology, Chinese Academy of Medical Sciences and Peking Union Medical College, Beijing 100730, People's Republic of China. ${ }^{4}$ Anesthesiology Department, Beijing Hospital, National Center of Gerontology, No.1 Dahua Road, Dong Dan, Beijing 100730, People's Republic of China. ${ }^{5}$ Department of Cardiology, Beijing Hospital, National Center of Gerontology, No.1 Dahua Road, Dong Dan, Beijing 100730, People's Republic of China. ${ }^{6}$ Pneumology Department, Beijing Hospital, National Center of Gerontology, No.1 Dahua Road, Dong Dan, Beijing 100730, People's Republic of China. ${ }^{7}$ Neurology Department, Beijing Hospital, National Center of Gerontology, No.1 Dahua Road, Dong Dan, Beijing 100730, People's Republic of China. ${ }^{8}$ The Fifth Ward of Ophthalmology Department, Shenzhen Eye Hospital, Shenzhen 518040, People's Republic of China.

Received: 22 January 2019 Accepted: 22 April 2019

Published online: 01 May 2019

\section{References}

1. Stone NJ, Robinson JG, Lichtenstein AH, Bairey MCN, Blum CB, Eckel RH, Goldberg AC, Gordon D, Levy D, Lloyd-Jones DM, et al. 2013 ACC/AHA guideline on the treatment of blood cholesterol to reduce atherosclerotic cardiovascular risk in adults: a report of the American College of Cardiology/ American Heart Association task force on practice guidelines. J Am Coll Cardiol. 2014;63:2889-934.

2. Bowman L, Hopewell JC, Chen F, Wallendszus K, Stevens W, Collins R, Wiviott SD, Cannon CP, Braunwald E, Sammons E, Landray M. Effects of Anacetrapib in patients with atherosclerotic vascular disease. N Engl J Med. 2017;377:1217-27.

3. JJK K, Cheng RX, Yap Y, Haldane V, Tan YG, KWQ T, Srivastava A, Ong PS, Perel $\mathrm{P}$, Legido-Quigley $\mathrm{H}$. Access and adherence to medications for the primary and secondary prevention of atherosclerotic cardiovascular disease in Singapore: a qualitative study. Patient Prefer Adherence. 2018;12:2481-98.

4. Dzau VJ. Atherosclerosis and hypertension: mechanisms and interrelationships. J Cardiovasc Pharmacol. 1990;15(Suppl 5):S59-64.

5. Rafieian-Kopaei M, Setorki M, Doudi M, Baradaran A, Nasri H. Atherosclerosis: process, indicators, risk factors and new hopes. Int J Prev Med. 2014;5:927-46.

6. Chrysohoou C, Kollia N, Tousoulis D. The link between depression and atherosclerosis through the pathways of inflammation and endothelium dysfunction. Maturitas. 2018;109:1-5.

7. Maeda M, Yamamoto I, Fujio Y, Azuma J. Homocysteine induces vascular endothelial growth factor expression in differentiated THP-1 macrophages. Biochim Biophys Acta. 2003;1623:41-6.

8. Wu N, Li RQ, Li L. SOAT1 deficiency attenuates atherosclerosis by regulating inflammation and cholesterol transportation via HO-1 pathway. Biochem Biophys Res Commun. 2018;501:343-50.

9. Higashi Y, Sukhanov S, Shai SY, Danchuk S, Tang R, Snarski P, Li Z, LobelleRich P, Wang M, Wang D, et al. Insulin-like growth Factor-1 receptor deficiency in macrophages accelerates atherosclerosis and induces an unstable plaque phenotype in apolipoprotein E-deficient mice. Circulation. 2016;133:2263-78.

10. Wang $X Q$, Liu ZH, Xue L, Lu L, Gao J, Shen Y, Yang K, Chen QJ, Zhang RY, Shen WF. C1q/TNF-related protein 1 links macrophage lipid metabolism to inflammation and atherosclerosis. Atherosclerosis. 2016;250:38-45.

11. Chen HH, Keyhanian $\mathrm{K}$, Zhou X, Vilmundarson RO, Almontashiri NA, Cruz SA, Pandey NR, Lerma YN, Ho T, Stewart CA, et al. IRF2BP2 reduces macrophage inflammation and susceptibility to atherosclerosis. Circ Res. 2015;117:671-83.

12. Yan D, Oho AUID, Zhang D, Lu L, Qiu H, Wang J. Vascular endothelial growth factor-modified macrophages accelerate reendothelialization and attenuate neointima formation after arterial injury in atherosclerosis-prone mice. J Cell Biochem. 2019.

13. Zhou $Y$, Zheng $X$, Chen $L$, Xu B, Jiang JT. microRNA-181b \resses the metastasis of lung cancer cells by targeting sex determining region $Y$ related high mobility group-box 6 (Sox6). Pathol Res Pract. 2018.

14. Ma J, Li H. The role of gut microbiota in atherosclerosis and hypertension. Front Pharmacol. 2018:9:1082.

15. Glass CK, Witztum JL. Atherosclerosis the road ahead. Cell. 2001;104:503-16. 
16. Mo C, Yang M, Han X, Li J, Gao G, Tai H, Huang N, Xiao H. Fat mass and obesity-associated protein attenuates lipid accumulation in macrophage foam cells and alleviates atherosclerosis in apolipoprotein E-deficient mice. J Hypertens. 2017;35:810-21.

17. Wang $H$, Liu D, Zhang $H$. Investigation of the underlying genes and mechanism of macrophage-enriched ruptured atherosclerotic plaques using bioinformatics method. J Atheroscler Thromb. 2019.

18. Ramon-Vazquez A, de la Rosa JV, Tabraue C, Lopez F, Diaz-Chico BN, Bosca L, Tontonoz P, Alemany S, Castrillo A. Common and differential transcriptional actions of nuclear receptors LXRalpha and LXRbeta in macrophages. Mol Cell Biol. 2019.

19. Qian Z, Li Y, Fan X, Zhang C, Wang Y, Jiang T, Oho AUID, Liu X. Prognostic value of a microRNA signature as a novel biomarker in patients with lowergrade gliomas. J Neuro-Oncol. 2018;137:127-37.

20. Li L, Lei Q, Zhang S, Kong L, Qin B. Screening and identification of key biomarkers in hepatocellular carcinoma: evidence from bioinformatic analysis. Oncol Rep. 2017;38:2607-18.

21. Cheng W, Ren X, Zhang C, Cai J, Liu Y, Han S, Wu A. Bioinformatic profiling identifies an immune-related risk signature for glioblastoma. Neurology. 2016;86:2226-34

22. Trychta KA, Back S, Henderson MJ, Harvey BK. KDEL receptors are differentially regulated to maintain the ER proteome under calcium deficiency. Cell Rep. 2018;25:1829-1840.e6.

23. Wang M, Kaufman RJ. Protein misfolding in the endoplasmic reticulum as a conduit to human disease. Nature. 2016;529:326-35.

24. Gao ZF, Ji XL, Gu J, Wang XY, Ding L, Zhang H, AUID- Oho: microRNA-107 protects against inflammation and endoplasmic reticulum stress of vascular endothelial cells via KRT1-dependent notch signaling pathway in a mouse model of coronary atherosclerosis. J Cell Physiol 2018.

25. Li G, Yin Q, Ji H, Wang Y, Liu H, Jiang L, Zhu F, Li B. A study on screening and antitumor effect of CD55-specific ligand peptide in cervical cancer cells. Drug Des Devel Ther. 2018;12:3899-912.

26. Loveless S, 0000-0002-5124-4115 AO, Neal JW, Howell OW, Harding KE, Sarkies P, Evans R, Bevan RJ, Hakobyan S, Harris CL, et al: tissue microarray methodology identifies complement pathway activation and dysregulation in progressive multiple sclerosis. Brain Pathol 2018, 28:507-520.

27. Trendelenburg M, Stallone F, Pershyna K, Eisenhut T, Twerenbold R, Wildi K, Dubler D, Schirmbeck L, Puelacher C, Rubini GM, et al. Complement activation products in acute heart failure: potential role in pathophysiology, responses to treatment and impacts on long-term survival. Eur Heart J Acute Cardiovasc Care. 2018;7:348-57.

28. Badiner N, Taylor SP, Forlenza K, Lachman RS, Bamshad M, Nickerson D, Cohn DH, Krakow D. Mutations in DYNC2H1, the cytoplasmic dynein 2, heavy chain 1 motor protein gene, cause short-rib polydactyly type I, Saldino-Noonan type. Clin Genet. 2017;92:158-65.

29. Mohammadi A, Vahabzadeh Z, Jamalzadeh S, Khalili T. Trimethylamine-Noxide, as a risk factor for atherosclerosis, induces stress in J774A.1 murine macrophages. Adv Med Sci. 2018;63:57-63.

Ready to submit your research? Choose BMC and benefit from:

- fast, convenient online submission

- thorough peer review by experienced researchers in your field

- rapid publication on acceptance

- support for research data, including large and complex data types

- gold Open Access which fosters wider collaboration and increased citations

- maximum visibility for your research: over $100 \mathrm{M}$ website views per year

At $\mathrm{BMC}$, research is always in progress.

Learn more biomedcentral.com/submissions 